P-65

\title{
Plastid DNA sequence diversity in a worldwide set of grapevine cultivars (Vitis vinifera $\mathbf{L}$. subsp. vinifera)
}

B. Schaal ${ }^{1}$, J. Beck ${ }^{1}$, S-C. Hsu ${ }^{1}$, T. Beridze ${ }^{2,3}$, M. Gamkrelidze ${ }^{2}$, M. Gogniashvili ${ }^{2}$, I. Pipia ${ }^{2}$, V. Tabidze $^{2}$, P. This ${ }^{4}$, R. Bacillieri', V. Gotsiridze ${ }^{5}$, M. Glonti $^{5}$

${ }^{1}$ Washington University, St. Louis, Missouri; ${ }^{2}$ Durmishidze Institute of Biochemistry and Biotechnology, Tbilisi, Georgia; ${ }^{3}$ Ilia State University, Tbilisi, Georgia $;{ }^{4}$ Institut National de la Recherche Agronomique (INRA) Montpellier, France ; ${ }^{6}$ Institute of Horticulture, Viticulture and Oenology, Tbilisi, Georgia

*Corresponding author: tgberidze@yahoo.com, tengiz_beridze@iliauni.edu.ge

DNA sequence diversity was investigated at two plastid regions (the $t r n H-p s b A$ intergenic spacer and the rpll6 intron) in a geographically diverse group of 113 cultivated grape samples. This group included 40 samples from the Republic of Georgia, home to over 500 grape cultivars and the earliest archaeological evidence of grape domestication. The greater Caucasus region in which Georgia lies is widely believed to be the area in which grape domestication began, and the study of genetic diversity in this region is viewed as key to understanding grape domestication in general. Four plastid haplotypes are evident in the 113 samples, and are designated by their character-states at each of the 3 polymorphic positions: (AAA)-23 samples, (ATT)-29 samples, (GTA)-34 samples, and (ATA)-27 samples. The AAA haplotype was only observed in Georgian samples, and these 23 "Rkatsiteli" group cultivars originate mostly from eastern Georgia.

Contrast this group with the nine Georgian cultivars (23\%) of the "Chkhaveri-Pinot noir" group (GTA), most of which are cultivated in western Georgia near the Black Sea coast. The observation that the Georgian cultivars exhibited both unique plastid DNA variation (the AAA haplotype) and all other observed plastid haplotypes is consistent with previous studies that have observed both unique and high levels of genetic variation in wild grape ( $V$. vinifera subsp. sylvestris) in the greater Caucasus region. 